\title{
The next of kin experiences of symptoms and distress among patients with colorectal cancer: diagnosis and treatment affecting the life situation
}

Karolina Ekholm, Carolin Grönberg, Sussanne Börjeson and Carina Berterö

\author{
Linköping University Post Print
}

\section{Tweet}

N.B.: When citing this work, cite the original article.

Original Publication:

Karolina Ekholm, Carolin Grönberg, Sussanne Börjeson and Carina Berterö, The next of kin experiences of symptoms and distress among patients with colorectal cancer: Diagnosis and treatment affecting the life situation, 2013, European Journal of Oncology Nursing, (17), 2, 125-130.

http://dx.doi.org/10.1016/j.ejon.2012.05.003

Copyright: Elsevier

http://www.elsevier.com/

Postprint available at: Linköping University Electronic Press

http://urn.kb.se/resolve?urn=urn:nbn:se:liu:diva-90610 


\section{The next of kin experiences of symptoms and distress}

among patients with colorectal cancer:

\section{Diagnosis and treatment affecting the life situation}

Ekholm K ${ }^{\mathrm{a}}$, Grönberg $\mathrm{C}^{\mathrm{a}}$, Börjeson $\mathrm{S}^{\mathrm{b}}$, Berterö $\mathrm{C}^{\mathrm{b}}$

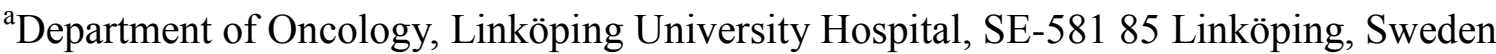

${ }^{b}$ Department of Medical and Health Sciences, Division of Nursing Science,

Faculty of Health Sciences, Linköping University, SE-581 85 Linköping, Sweden

karolina.ekholm@lio.se; carolin carolin.gronberg@lio.se; Sussanne.Borjeson@liu.se;

carina.bertero@liu.se

\section{Corresponding Author;}

Carina Berterö, RNT, BSc, MScN, PhD, Professor

Department of Medical and Health Sciences

Division of Nursing Science

Faculty of Health Sciences $\quad$ Tel +46101037768

SE-581 85 Linköping $\quad$ Fax +4613123285

SWEDEN

e-mail: carina.bertero@liu.se 


\begin{abstract}
Purpose. To identify symptoms/distress among patients with colorectal cancer undergoing chemotherapy, from the viewpoint of the next of kin, and to establish whether there are any barriers to reporting these problems.
\end{abstract}

Methods. Individual face-to-face interviews with fourteen next of kin were conducted. Qualitative content analysis was used to analyse the transcripts.

Results. Three areas were identified: symptoms presented, barriers to reporting symptoms/distress, and influences on life for the next of kin. Nine symptoms were raised as most common by the next of kin. Almost all the next of kin denied that they had experienced any barriers to reporting symptoms/distress but some did exist, namely barriers to proper communication and barriers of time. The next of kin made another interpretation of barriers; they did not interpret it as hinder or obstacle. All next of kin talked to a large extent about how the patient's disease and treatment affected them as next of kin. It affected them psychologically, they had to revaluate their life, and it influenced their social life.

Conclusions. The symptoms reported during chemotherapy were similar to those found in other studies on patients. Barriers to reporting symptoms were mentioned, but not to a great extent. Although it was not the main purpose of the study, the next of kin raised concerns about the patient's disease and treatment and how it influenced next of kin life.

Keywords: Colorectal cancer, next of kin, influences on life, symptoms, barriers, qualitative content analysis 


\section{Introduction}

Cancer affects the whole family, not only the patient. People living close to patients with cancer report having psychological disorders in the same way as the patients do (Drabe et al, 2008; Edvardsson and Ahlström, 2008; Sjövall et al, 2010). The next of kin is defined by the patient as the person closest to him/her; a family member or a friend (Doyle, 2004). Living with a person with cancer means a changed life situation for the next of kin, and leads to changed routines and roles in the family (Edvardsson and Ahlström, 2008; Esbensen and Thomé, 2010; Sjövall et al, 2010; Steinvall et al, 2011). Sometimes patients change due to their disease, which can lead to conflicts. It can be hard to accept that the patient is tired and does not have the same strength. The next of kin has to deal with their own new role in the relationship (Esbensen and Thomé, 2010). Next of kin state that it is important that life continues as normally as possible, and describe friends and other people around them as important to maintaining normality (Esbensen and Thomé, 2010; Sjövall et al, 2010; Steinvall et al, 2011). It has been described that the next of kin and the patient revaluate their life together and prioritise things in life differently to before the diagnosis (Esbensen and Thomé, 2010; Steinvall et al, 2011). Hence, next of kin are important for the patient during the entire disease process, and also often provide information to health care professionals about the cancer patient (Aaronson, 1991; Lobchuck and Degner, 2002), and they need support to manage their new life situation. Supporting the next of kin benefits the patient and the family members (McPehrson et al, 2008). Due to different experiences of the situation, symptoms and distresses may be perceived and articulated differently by the patient, the next of kin and the health care professionals (Broberger et al, 2005; Sneeuw et al, 1999; Miakowski et al, 1997). Next of kin are more accurate when identifying concrete observable symptoms i.e. vomiting, rather than subjective symptoms such as pain, psychological status and symptom distress (Lobchuck and Degner, 2002; Tang and McCorkle, 2002). For example; there is a poor level of agreement between patient and family when judging pain (Lin, 2001). Levels of 
agreement for individual symptoms range from poor to very good, and there is a tendency for caregivers to overestimate the importance of psychological symptoms compared to physical symptoms (McPehrson et al, 2008).

In this study, the focus is on colorectal cancer (CRC), the third most common type of cancer in the world (Grenon and Chan, 2009; IARC, 2010; Labianca et al, 2010; Wilkes and Hartshorn, 2009). CRC is more common in industrial countries such as the USA, Europe, Australia and New Zealand (Oncology Centre, 2008). Early stage CRC does not often present symptoms or gives non-specific symptoms such as changed bowel habits and abdominal discomfort. Specific symptoms for CRC appear later during the disease and can include, for example, changed toilet routines, abdominal pain, nausea, vomiting and bleeding when visiting the toilet (Labianca et al, 2010).

The primary treatment for CRC is surgery. Adjuvant chemotherapy for CRC in stages II and III is given in the form of FU/LV in different combinations with additional chemotherapy (oxaliplatin, irinotecan and capecitabine) and/or target agents (cetuximab, panitumumab and bevacizumab). This often complex and long-lasting treatment gives side effects. The side effects of chemotherapy vary depending on the agents used: myelosuppression, nausea, diarrhoea, neurotoxicity and hand-foot syndrome have been reported (Berger et al, 2010; Hallquist Viale and Sommers, 2007). The worst and most distressing side effect reported of chemotherapy for patients with CRC is fatigue (Berger et al, 2010; Henry et al, 2008; Kearney et al, 2008, Stone et al, 2000).

This study is a part of a project which aimed to investigate symptoms/distress and barriers to reporting these among patients with $\mathrm{CRC}$, from three perspectives; the patient, the next of kin and the hospital staff. The result of research on hospital staff indicated that the next of kin may experience barriers to reporting symptoms (Börjeson et al, 2011). For patients with 
$\mathrm{CRC}$, both the disease and the treatment cause troublesome symptoms and side effects

(Börjeson et al, 2011; Knowles et al, 2007). To the best of our knowledge there are no studies that, from the next of kin's point of view, focus on the patient's symptoms and side effects of chemotherapy. In addition, no studies have been found whose focus is on possible barriers to reporting symptoms. According to earlier studies, the patient's disease influences the next of kin and their life (Drabe et al, 2008; Edvardsson and Ahlström, 2008; Sjövall et al, 2010). Therefore, the aim of this study was to identify symptoms/distress among patients with colorectal cancer undergoing chemotherapy, from the viewpoint of the next of kin, and to establish whether the next of kin experience any barriers to reporting these problems.

\section{Methods}

\section{Setting and sample}

The research was a part of a larger study carried out at two hospitals in southern Sweden, where patients were invited to respond to several questionnaires and take part in interviews. In this larger study, both the next of kin and professionals were interviewed. In the part presented here the participants were next of kin to CRC patients. The inclusion criteria for the study were (a) being older than 18 years of age, (b) being identified as a next of kin by a patient receiving FOLFOX, Xeloda or XELOX chemotherapy for CRC, and (c) being willing to share experiences with us. Purposive sampling was used in order to recruit informative participants, since they had experiences of being a next of kin to a person with CRC undergoing treatment (Patton, 2002). Patients were identified by the responsible physician together with the process leader of the study (a nurse involved in the project at each clinic). Every fourth next of kin to the patients involved (110 patients) was informed and requested, both orally and in written form, to participate in the project, and the same information was given to the next of kin. The recruitment continued for one year. Expectations were a sample of 15 to 25 next of kin. Informed consent was required prior to participation. The interviews 
with next of kin were conducted after the second chemotherapy session for patients who were treated with a 2-week treatment course with XELOX or Xeloda, and for patients who received treatment with the FOLFOX, 2-day treatment; the interviews with their next of kin took place after the third chemotherapy session. This procedure ensured that all patients had received a similar volume intensity of chemotherapy at the time of interview with the next of kin. The study was approved by the regional Ethical Review Board in Linköping, and the next of kin and the patients gave their written consent before entering the study.

\section{Data Collection}

Data were collected using qualitative face-to-face interviews. The interviews were conducted at a place chosen by the next of kin, some in their home, and some at a consultation room at the hospital. An interview guide was used consisting of two domains. Every interview started with an open question in which the next of kin were asked to describe the patient's disease and symptoms and mention if there were any barriers to reporting symptoms. The interview continued with further questions to make things clearer (Patton, 2002). Two interviewers carried out all interviews. They were skilled in interviewing and applied the same approach. Each interview took about 60 minutes (median 40 minutes), was audio-taped, and then transcribed verbatim. Before the interviews there was some small talk, and after the interviews there was some reflection upon the interview situation.

\section{Data analysis}

In order to capture the next of kin experiences, qualitative content analysis was used to analyse the transcripts (Hsieh and Shannon, 2005; Patton, 2002). This was a continuous process of carefully and thoroughly reading and re-reading the transcripts, being vigilant for variations in data and searching for patterns. After attentive reading and with the research questions in mind, three areas emerged. For the first area, directed content analysis was used. 
Segments of text were sorted according to symptoms, which became the categories. For the other two areas, conventional content analysis with interpretation was used. The analysis started with coding, in which patterns and segments of text that captured key concepts were identified. One interview after another and one area at a time were analysed in this way. The coding were made by the two first authors independently and then further analysis and discussions ensued until agreement was reached. There was strong agreement between the researchers. The codes were sorted into categories based on their content and meaning. The names of the categories were developed from interpretation of the data (Hsieh and Shannon, 2005). In order to increase the trustworthiness of the findings, the analysis was subjected to peer-debriefing and was continuously scrutinized by two additional researchers who gave their viewpoint, and discussions were held during the process (Hsieh and Shannon, 2005).

\section{Results}

Fourteen next of kin were interviewed. The informants were eight wives, five husbands and one son. They were aged between 25-70 years.

The analysis identified three areas; symptoms presented, influences on life for the next of kin, and barriers to reporting symptoms/distress.

\section{Symptoms presented}

Nine symptoms were mentioned by the next of kin as being the most common among patients with CRC before and after chemotherapy. These symptoms were clearly expressed and described. While directed content analysis was used in this part, the next of kin descriptions were as follows (we have not interpreted conditions such as hand-and-foot syndrome etc. as neurotoxic side-effects). We have also presented how many next of kin mentioned the symptoms. 


\section{Fatigue}

Fatigue was frequently mentioned both before (9/14) and after chemotherapy (11/14). The patients were more tired than usual. They could not manage to do things they had done previously, and sometimes had a hard time getting out of bed. After chemotherapy almost all patients were or looked tired. Fatigue was described as not having the power to do things in the same way as before. Some said that the grade of the patient's fatigue was prominent; from hardly being able to go to the toilet some days, to falling asleep while watching TV. This tiredness seemed to influence the patient's ability to concentrate and affected the patient's mood.

\section{Intestinal disorders}

This was the most common symptom before chemotherapy (11/14) reported by the next of kin. Symptoms reported were stomach pain, stomach spasms, a swollen feeling in the stomach, constipation problems and blood in the faeces, which the patient thought was due to haemorrhoids. During chemotherapy, intestinal disorders were still a problem for some (6/14), constipation and diarrhoea were the most common symptoms mentioned even though problems with sudden stabbing pains in the stomach and swelling of the stomach could appear.

\section{Nausea}

Half of the next of kin (7/14) reported that the patients had problems with nausea after chemotherapy, while only one reported this before chemotherapy. The nausea varied from being mild to severe with vomiting, and increased with every treatment. Some (5/14) reported that the patient had problems with taste changes after chemotherapy. Food did not taste like it used to and it changed from one day to another. 


\section{Uneasiness}

Before starting chemotherapy some (5/14) mentioned that the patient was changed somehow. After surgery the patients were more labile and sensitive to touch. During chemotherapy many $(9 / 14)$ thought the patient had experienced some sort of psychological effect. They described patients as being nervous, worried, irritated, crying more easily, being depressed, having a hard time relaxing and talking with close relatives, having a fluctuating temper, and not being themselves. The anxiety interrupted the patients' sleep, especially before going to the hospital for a new cycle of chemotherapy.

\section{Hand-foot-syndrome}

Pricking sensations in the hands and feet that started almost immediately after chemotherapy were frequently reported (8/14). According to the next of kin, the patients had problems holding cold things in their hands and had to wear gloves when fetching things from the refrigerator. A general sensitivity to cold things was mentioned, as well as dry and fissured hands.

\section{Pain}

Pain before chemotherapy was mentioned by a few (4/14) next of kin. The pain was described as the patient having pain in the back and over the kidneys. Pain on one side of the throat down to the shoulder was also mentioned. Only one patient had pain in the stomach after chemotherapy, according to the next of kin.

\section{Body changes}

A few next of kin mentioned that the patient lost some weight before (3/14) and after chemotherapy (2/14). During chemotherapy, some noticed significant loss of muscle and 
condition in the patient, darker and coarser hair, alopecia, and spots on the face of the patient after being outdoors.

\section{Mouth problems}

Some next of kin reported the patient had problems with the mouth after chemotherapy (4/14). Difficulties eating cold food and drink and an irritated feeling in the mucous membranes in the mouth were mentioned. The next of kin noticed that these symptoms were worse three to four days after chemotherapy.

\section{Allergic reaction}

A few $(2 / 14)$ said that the patients had fever and an allergic reaction in connection with chemotherapy, with nettle rash and a swollen face and tongue. Because of these problems, the patient had to end chemotherapy treatment. Allergic reactions many days after chemotherapy had been given were also reported.

\section{Barriers to reporting symptoms/distress}

Almost all next of kin denied that they had experienced barriers to reporting symptoms/distress. Despite this they mentioned barriers to proper communication, situations when they felt the hospital staff did not have enough time, and difficulties with some tasks.

\section{Barriers to proper communication and individual information}

Many next of kin (9/14) expressed lack of information, which made them feel unsure and frustrated. They described situations when they were uncertain how to act and when they were unsure whether the patient's symptoms were caused by chemotherapy or not. They felt they did not get correct and concrete answers to all their questions, " I felt that I actually didn't get any answer, they just said that one has to expect that one is feeling sick for a 
couple of days" (J 16). They were unsure whether the doctor or the nurse should answer their questions, and instead, they searched for information on the Internet. A feeling of being forgotten and not knowing where to phone for support was expressed. Due to these feelings, several expectations and self-made explanations for the patient's symptoms appeared. Fatigue was explained as natural, caused by advanced age rather than by the disease or chemotherapy, and the side effects of chemotherapy were expected to be worse than they were "You had almost expected worse problems, you hear so much from others that they got sick and everything” (L 4).

\section{Barriers of time}

Few (4/14) said that they did not raise all their concerns with the nurse or the doctor because they thought the hospital staff had so much to do. Chemotherapy treatment was experienced as an assembly line; "but these kinds of questions maybe need a longer answer... and the question is if she has enough time when there are so many patients to treat" (L 7).The feeling of being a troublemaker and wasting the hospital staff's time was expressed, and so only the most important questions were asked.

\section{Troublesome tasks}

Things that were hard to talk about were mentioned by a few of the next of kin (4/14). One thing they brought up was that the patient was afraid of going to the hospital, "He is very afraid or.., has been previously anyhow, before every visit to the hospital” (J 10). Reduced sexual desire, loss of sexual activity and psychological changes were also mentioned as hard to talk about.

\section{Influences on life for the next of kin}

Although the next of kin were asked about the patient's symptoms/distress and if there were 
any barriers to reporting these, all the next of kin spoke very much about how the patient's disease and treatment had affected them. Five components were identified and interpreted in this area.

\section{Psychological influence and burden}

Almost all the next of kin (13/14) were concerned about the patient. They said it was tough, they were worried and sad. They were concerned about whether the patient would survive. Even though they tried not to think about it all the time, suddenly the thoughts were there again. "You think about it all the time...//...it's never gone from my thoughts " (L 28). There were feelings of fear; they were scared and thought that something bad would happen with the patient. They mentioned that patients could be reluctant to take their drugs, a situation described as more demanding for the next of kin than for the patient. A changed daily life with new routines and instructions concerning the treatment were reported. The next of kin had many speculations, feelings and thoughts about the patient's disease. They felt frustrated when they could not get the chance to talk with the doctor alone.

\section{Re-evaluation of life and relationship}

Half of the next of kin (7/14) said the disease had changed their lives and that the situation caused insecurity. Their lives changed in that they got closer to the patient and they prioritized other things in life "it reminds you that you will not have each other forever.... and you think about time in a different way to before..." (L 10). They focused on living as good a life as possible, and their families and relatives became more important. They seized their days differently, and did not take anything for granted. Some described their lives as circling around the treatment. Few next of kin $(2 / 14)$ mentioned that their sexual life had altered; their sexual activity had stopped due to the absence of desire from the patients and there was no energy for sexual activities "I don't know why, but there is no desire" (L 13). 


\section{Influence on social life}

Almost half of the next of kin (6/14) mentioned that the disease had influenced their social life. They felt a little unsociable, not seeing friends as much as before because the patient felt tired. The next of kin described how they held themselves back in life. They described taking into account the chemotherapy treatment before planning a vacation; "We'll have to take it between the treatments, if he copes with it" (J 22).

\section{Acceptance}

A few next of kin (4/14) talked about how they had resigned themselves to the situation. They felt they did not have a choice and accepted that the patient sometimes had a varied temperament due to the disease and treatment, "You see, one tries to change it a little to accept the situation" (J 19). Frustration over the situation changed into acceptance.

\section{Not prioritising themselves}

The feeling of not being able to leave the house and go for a walk as they used to do was reported by some (4/14). They felt that they had to be home, checking on the patient. The next of kin mentioned that they had to change their sleeping routines due to the patient's disease. The patients were prioritised; they needed to sleep on their own in order to sleep well.

\section{Discussion}

The aim of this study was to identify symptoms/distress, among patients with CRC undergoing chemotherapy, as perceived by the next of kin, and to establish whether there were any barriers to reporting these problems. We found fatigue, nausea, intestinal disorders and hand-foot syndrome to be the most common symptoms reported by next of kin. This is 
confirmed by other studies investigating patients (Aprile et al, 2008; Berger et al, 2010; Goldberg et al, 2007; Griffin-Sobel, 2006; Hallquist Viale and Sommers, 2007; Henry et al, 2008; Kearney et al, 2008). However, the next of kin reported that nausea worsened with every treatment, whereas other studies on patients, in contradiction, have found that nausea decreases after every treatment (Henry et al, 2008; Kearney et al, 2008). The next of kin mentioned that the patients were psychologically changed. This confirms previous findings showing that patients may experience fear, be depressed, suffer from anxiety and many change their normal roles (Cotrim and Pereira, 2008; Griffin-Sobel, 2006).

Next of kin denied that they had experienced any barriers to reporting symptoms, but our analyses showed situations where they said that they did not know what to report and/or did not report everything to the nursing staff. Barriers to proper communication and individual information were mentioned. Next of kin were not satisfied with information given, and there was a difference in the information wanted by the patient and by the next of kin (Northouse et al, 1999). The patient and the next of kin did not necessarily need the same questions to be answered (Feldman-Stewart et al, 2001). Information on non-medical topics such as the impact of cancer on relationships was more likely to be lacking than medically-oriented information (Adams et al, 2009). In our study, the next of kin said that they chose to report to the hospital staff only a few selected symptoms that they thought were the most important. Somehow they got the feeling that the hospital staff were short of time, and therefore some questions were not asked.

One of our main findings was that the patients' disease influenced the next of kin to a great extent. Next of kin frequently reported that the patient's disease and treatment affected them in many ways. Almost all expressed this as a psychological burden; they mentioned a changed daily life, new routines and changed roles. Similar results are found in other studies (Esbensen and Thomé, 2010; Northouse et al, 1999; Steinvall et al, 2011). The next of kin 
described a constant anxiety about the patient, and studies have shown that the next of kin report more emotional distress than the patients do (Northouse et al,1999; Northouse et al, 2000). Also, health care use by next of kin increases as well as diagnoses of psychiatric problems (Sjövall et al, 2009), and in general they have an increase in sick leave (Sjövall et al, 2010). This psychological burden makes next of kin more vulnerable and actually sick. In our study, the next of kin said that their attitude towards life had changed and they felt closer to their sick relative. They did not take the patient for granted, and their families and close friends had become more important. At the same time, we found that they felt unsociable. This is in agreement with another study reporting that a feeling of isolation was described by the next of kin (Steinvall et al, 2011). Other studies show that the disease changes their way of looking at life, and they revaluate what is important (Edvardsson and Ahlström, 2008; Steinvall et al, 2011). Some become resigned to the situation and this is difficult for the next of kin who are supposed to be the healthy and strong ones. Studies show that next of kin keep their feelings and anxiety to themselves; they do not want to worry the patient (Esbensen and Thomé, 2010; Steinvall et al, 2011). This means that the next of kin do not make taking care of themselves a priority and thus are likely to get sick. It is of the greatest importance that next of kin receive support in this demanding situation.

A strength of this study is that we analysed the interview transcripts with both direct and conventional content analysis, which gave varied data (Hsieh and Shannon, 2005). The methods also have weaknesses; when direct content analysis is used there is a risk that the researcher will find evidence that supports the theory, which can blind the researcher. We tried to avoid that by analysing the transcripts first on our own (KE, CG) and then together. With conventional analysis it is a challenge to develop a complete understanding of the context and to find the key categories. By reading the transcripts many times, analysing each one separately, and then coming to consensus through discussion we have minimized this. 
Two additional researchers reviewed the analysis to increase the trustworthiness. The next of kin that were interviewed were predominantly wives or husbands. This strengthens the study, because those who live closest to the patient are the ones that can give us the most complete information. Most of the patients had colon cancer but in our article we use CRC, which also includes rectal cancer. That could be a weakness since the two diagnoses can differ in treatment and symptoms. However, CRC is a commonly used term and colon cancer has a higher incidence than rectal cancer (Oncology Centre, 2008; Wilkes and Hartshorn, 2009). Another limitation of this study could be that the next of kin did not put their focus on symptoms and barriers. Instead they focused on how they were affected by having CRC in the family. This was not the aim of the study but was nevertheless an interesting and important finding. Why did this happen? It could be that the next of kin often feel slighted, since attention is on the patient, the treatment etc. In this interview they had a person willing to listen to them for one hour, without interrupting them.

The next of kin are important for the patient (Steinvall et al, 2011), and a cancer diagnosis influences the whole family (Northouse et al, 2000).

It is therefore of the highest priority for healthcare providers to pay attention to the next of kin, giving them opportunities to talk in private about their feelings and thoughts concerning the disease and treatment. Health care providers need to improve and individualize the information we provide to the next of kin. In order to achieve this, a care navigator following the patient and the next of kin through the cancer trajectory would be a good form of support.

\section{Conclusions}

In this study, the next of kin were asked to identify symptoms/distress among patients with $\mathrm{CRC}$ receiving chemotherapy, and barriers to reporting these problems. The symptoms reported are the same, on the whole, as those identified in other studies made on patients, but 
research is needed in the area of hand-foot syndrome. The next of kin mentioned barriers to reporting symptoms, but not to a large extent. Mostly they discussed how the patient's disease and treatment had psychologically affected them. It was a psychological burden that influenced their life in many ways. Further research is necessary in order to investigate the needs of the next of kin.

\section{Conflict of interest}

There is no financial relationship to FORSS who supported us with funding. As authors we have full control of all primary data and agree the journal to review this data if requested.

\section{Acknowledgement}

We thank the next of kin for participating and sharing their experiences with us. We also thank other members of the research team planning the large project that this study is part of; Gunilla Pettersson, MonicaRösliden, CeciliaBladh, SusanneBorén, Viktoria Magnusson, MitraUnosson and HansStarkhammar.

We are also grateful to the Department of Oncology, Linköping University Hospital, the Medical Research Council of Southeast Sweden and the Division of Nursing Science at the Department of Medical and Health Sciences for financial support.

\section{References}

Adams E, Boulton M, Watson E. 2009.The information needs of partners and family members of cancer patients: A systematic literature review. Patient Education \& Counseling 77(2), 179-186.

Aprile G, Ramoni M, Keefe D, Sonis S. 2008. Application of distance matrices to define associations between acute toxicities in colorectal cancer patients receiving chemotherapy. Cancer 112(2), 284-292.

Aaronson NK. 1991. Methodological issues in assessing the quality of life in cancer patients. 
Cancer 1(suppl),844-850.

Berger A, Grem J, Visovsky C, Marunda H, Yurkovich J.2010. Fatigue and Other Variables During Adjuvant Chemotherapy for Colon and Rectal Cancer. Oncology Nursing Forum37(6), E359-69.

Broberger E, Tishelman C, von Essen 1. 2005. Discrepancies and Similarities in how patients with Lung cancer and Their Professional and family Caregivers assess Symptom Occurrence and symptom Distress. Journal of Pain and Symptom Management 29(6), 572 582.

Börjeson S, Starkhammar H, Berterö C. 2011.Common Symptoms Experienced Among Patients with Colorectal Cancer, and Barriers to Reporting Symptoms or Distress: the Staff Perspective. Australian- Asian Journal of Cancer 10(1), 11-20.

Cotrim H, Pereira G. 2008. Impact of colorectal cancer on patient and family: implications for care. European Journal of Oncology Nursing 12(3), 217-226.

Drabe N, Zwahlen D, Buchi S, Moergeli H, Zwahlen R, Jenewein J. 2008.Psychiatric morbidity and quality of life in wives of men with long-term head and neck cancer. Psycho-Oncology 17(2), 199-204.

Doyle, D. Oxford Textbook of Palliative Medicine. New York: Oxford university press; 2004 Edvardsson T, Ahlstrom G. 2008. Being the next of kin of a person with a low-grade glioma. Psycho-Oncology 17(6), 584-591.

Esbensen B, Thomé B.2010. Being next of kin to an elderly person with cancer. Scandinavian Journal of Caring Sciences 24(4), 648-654.

Feldman-Stewart D, Brundage M, Mackillop W.2001. What questions do families of patients with early-stage prostate cancer want answered? Journal of Family Nursing 7(2), 188-207.

Goldberg R, Rothenberg M, Van Cutsem E, Benson I, Blanke C, Viele C, et al.2007. The Continuum of Care: A Paradigm for the Management of Metastatic Colorectal Cancer. Oncologist 12(1), 38-50. 
Grenon NN, Chan J.2009. Managing Toxicities Associated With Colorectal Cancer

Chemotherapy and Targeted Therapy: A New Guide for Nurses. Clinical Journal of Oncology Nursing 13(3),285-296.

Griffin-Sobel J.2006. Symptom management of advanced colorectal cancer. Surgical Oncology Clinics of North America 15(1),213-222.

Hallquist Viale P, Sommers R.2007. Nursing care of patients receiving chemotherapy for metastatic colorectal cancer: Implications of the treatment continuum concept. Seminars in oncology nursing 23(1),22-35.

Henry D, Viswanathan H, Elkin E, Traina S, Wade S, Cella D. 2008.Symptoms and treatment burden associated with cancer treatment: results from a cross-sectional national survey in the U.S. Supportive Care and Cancer 16(7), 791-801.

Hsieh H, Shannon S. 2005.Three approaches to qualitative content analysis. Qualitative Health Research 15(9), 1277-1288.

IARC. PDFs Online-World Cancer Report. World Cancer Report. 2008. (ED) Boyle P and Levin B. Available at: http://www.iarc.fr/en/publications/pdfsonline/wcr/2008/wcr_2008.pdf Accessed September 7th, 2011.

Kearney N, Miller M, Maguire R, Dolan S, MacDonald R, Wengstrom Y, et al. 2008. WISECARE+: results of a European study of a nursing intervention for the management of chemotherapy-related symptoms. European Journal of Oncology Nursing 12(5), 443 448.

Knowles G, Sherwood L, Dunlop M, Dean G, Jodrell D, Preston E, et al. 2007.Developing and piloting a nurse-led model of follow-up in the multidisciplinary management of colorectal cancer. European Journal of Oncology Nursing11(3), 212-223.

Labianca R, Beretta G, Kildani B, Milesi L, Merlin F, Wils J, et al. 2010.Colon cancer. Critical Reviews in Oncology/Hematology 74(2),106-133. 
Lin Chia-Chin. 2001. Congruity of Cancer Pain Perceptions Between Taiwanese Patients and Family Caregivers: Relationship to Patients' Concerns About Reporting Pain and Using Analgesics. Journal of pain and Symptom Management 21(1), 18-26.

Lobchuck MM, Degner LF. 2002. Patients With cancer and Next- of-Kin Response Comparability on Physical and Psychological Symptom Well-Being. Cancer Nursing 25(5), 358-374.

McPehrson CJ, Wilson KG, Lobchuk MM, Bratman S. 2008. Family Caregivers Assessment of symptoms in Patients with Advanced Cancer: Concordance with Patients and factors Affecting Accuracy. Journal of Pain and Symptom management 35(1), 70-82.

Miakowski C, Kargness L, Dibble S, WallhagenM. 1997. Differences in mood states, health status, and caregiver strain between family caregivers of oncology outpatients with and without cancer-related pain. Journal of Pain Symptom management 13, 138-147.

Northouse L, Mood D, Templin T, Mellon S, George T. 2000.Couples' patterns of adjustment to colon cancer. Social Science \& Medicine 50(2), 271-284.

Northouse L, Schafer J, Tipton J, Metivier L. 1999.The concerns of patients and spouses after the diagnosis of colon cancer: a qualitative analysis. Journal of Wound, Ostomy \& Continence Nursing 26(1), 8-17.

Oncology Centre (Onkologiskt centrum). 2008. Nationellt vårdprogram för kolorektal cancer. (National program for Colo Rectal Cancer care). Available at: http://www.ocsyd.se/VPverksamhet/Gastrointest \%20ca/Vardprogr_Kolorektal-cancer081120.pdf Accessed January $10^{\text {th }}, 2011$

Patton MQ. 2002. Qualitative research \& evaluation methods. 3.ed. SAGE publication, London.

Sjövall K, Attner B, Lithman T, Noreen D, Gunnars B, Olsson H, et al.2009. Influence on the health of the partner affected by tumor disease in the wife or husband based on a population-based register study of cancer in Sweden. Journal of Clinical Oncology 27(28), 
4781-4786.

Sjövall K, Attner B, Lithman T, Noreen D, Gunnars B, Englund M, et al. 2010. Sick leave of spouses to cancer patients before and after diagnosis. Acta Oncologica 49(4), 467-473.

Sneeuw KCA, Aaronson NK, Sprangers MAG, Detmar SB, Wever LDV, Schornage HA. 1999. Evaluating the quality of life of cancer patients: assessments by patients, significant others, physicians and nurses. British Journal of Cancer 81(1), 87-94.

Steinvall K, Johansson H, Berterö C. 2011.Balancing a Changed Life Situation: The Lived Experience from Next of Kin to Persons With Inoperable Lung Cancer. American Journal of Hospice \& Palliative Medicine 28(2), 82-89.

Stone P, Richardson A, Ream E, Smith AG, Kerr DJ, Kearney N. 2000.Cancer-related fatigue: inevitable, unimportant and untreatable? Results of a multi-centre patient survey, Cancer Fatigue Forum Annals of Oncology 11(8), 971-975.

Tang ST, McCorkle R. 2002. Use of family proxies in quality of life research for cancer patients at the end of life: a literature review. Cancer Investigation 20, 1086-1104. 
Table 1. Characteristics of the patients of which next of kin were interviewed

\begin{tabular}{llllll}
\hline Sex & Age* (years) & Social status & Occupation & Chemotherapy regime & Treatment stage \\
\hline Male & 37 & Married/cohabitant & on sick-leave & Xelox & Palliative \\
Female & 41 & Married/cohabitant & on sick-leave & Folfox & Neoadjuvant \\
Female & 46 & Married/cohabitant & on sick-leave & Folfox & Adjuvant \\
Male & 52 & Married/cohabitant & on sick-leave & Folfox & Neoadjuvant \\
Female & 55 & Married/cohabitant & Employee full & Xelox & Adjuvant \\
Female & 56 & Married/cohabitant & on sick-leave & Xelox & Adjuvant \\
Male & 59 & Married/cohabitant & Employee part & Folfox & Neoadjuvant \\
Female & 62 & Married/cohabitant & Disable. pension & Xelox & Adjuvant \\
Female & 63 & Married/cohabitant & Disable. pension & Folfox & Adjuvant \\
Male & 71 & Married/cohabitant & Retired & Xeloda & Neoadjuvant \\
Male & 73 & Married/cohabitant & Retired & Folfox & Neoadjuvant \\
Male & 76 & Married/cohabitant & Retired & Xelox & Adjuvant \\
Female & 76 & Married/cohabitant & Retired & Xeloda & Palliative \\
Age & 85 & married/cohabitant & Retired & Xeloda & Palliative \\
\hline The patients'age when the interview with the next of kin was carried out.
\end{tabular}

Age* $^{*}$ The patients' age when the interview with the next of kin was carried out. 